\title{
BMJ The beliefs and expectations of patients Open and caregivers about home haemodialysis: an interview study
}

\author{
Allison Tong, ${ }^{1,2}$ Suetonia Palmer, ${ }^{3}$ Braden Manns, ${ }^{4}$ Jonathan C Craig, ${ }^{1,2}$ \\ Marinella Ruospo, ${ }^{5}$ Letizia Gargano, ${ }^{5}$ David W Johnson, ${ }^{6}$ Jörgen Hegbrant, ${ }^{7}$ \\ Måns Olsson, ${ }^{8}$ Steven Fishbane, ${ }^{9}$ Giovanni F M Strippoli ${ }^{2,10,11,12}$
}

To cite: Tong A, Palmer S, Manns B, et al. The beliefs and expectations of patients and caregivers about home haemodialysis: an interview study. BMJ Open 2013;3: e002148. doi:10.1136/ bmjopen-2012-002148

- Prepublication history and additional material for this paper are available online. To view these files please visit the journal online (http://dx.doi.org/10.1136/ bmjopen-2012-002148).

Received 21 September 2012 Revised 22 November 2012 Accepted 26 November 2012

This final article is available for use under the terms of the Creative Commons Attribution Non-Commercial 2.0 Licence; see http://bmjopen.bmj.com

For numbered affiliations see end of article.

Correspondence to Professor Giovanni Strippoli; gfmstrippoli@gmail.com

\section{ABSTRACT}

Objectives: To explore the beliefs and expectations of patients and their caregivers about home haemodialysis in Italy where the prevalence of home haemodialysis is low.

Design: Semistructured, qualitative interview study with purposive sampling and thematic analysis.

Setting: Four dialysis centres in Italy without home haemodialysis services (Bari, Marsala, Nissoria and Taranto).

Participants: 22 patients receiving in-centre haemodialysis and 20 of their identified caregivers.

Results: We identified seven major themes that were central to patient and caregiver perceptions of home haemodialysis in regions without established services. Three positive themes were: flexibility and freedom (increased autonomy, minimised wasted time, liberation from strict dialysis schedules and gaining self-worth); comfort in familiar surroundings (family presence and support, avoiding the need for dialysis in hospital) and altruistic motivation to do home haemodialysis as an exemplar for other patients and families. Four negative themes were: disrupting sense of normality; family burden (an onerous responsibility, caregiver uncertainty and panic and visually confronting); housing constraints; healthcare by 'professionals' not 'amateurs' (relinquishing security and satisfaction with in-centre services) and isolation from peer support.

Conclusions: Patients without direct experience or previous education about home haemodialysis and their caregivers recognise the autonomy of home haemodialysis but are very concerned about the potential burden and personal sacrifice home haemodialysis will impose on caregivers and feel apprehensive about accepting the medical responsibilities of dialysis. To promote acceptance and uptake of home haemodialysis among patients and caregivers who have no experience of home dialysis, effective strategies are needed that provide information about home haemodialysis to patients and their caregivers, assure access to caregiver respite, provide continuous availability of medical and technical advice and facilitate peer patient support.

\section{ARTICLE SUMMARY}

\section{Article focus}

- This study aims to explore the beliefs and attitudes of patients and caregivers about home haemodialysis in a region with very low rates of home haemodialysis use.

\section{Key messages}

- Similar to patients on in-centre haemodialysis in other global regions, home haemodialysis is perceived as confronting and unsafe by patients on in-centre haemodialysis and their caregivers in regions without home haemodialysis services.

- Suggested strategies to increase the acceptability and uptake of home haemodialysis in regions without established services include educating patients about home haemodialysis as a treatment choice, assuring caregiver respite and providing round-the-clock technical and medical advice.

- Strategies that have been shown to improve uptake of home haemodialysis in other areas may be effective in regions establishing home haemodialysis programmes and warrant further evaluation.

Strengths and limitations of this study

- This is the first study to examine the expectations and beliefs about home haemodialysis among in-centre haemodialysis patients and their caregivers who have received no formal education, choice or experience of home haemodialysis.

- All interviews were conducted and coded in Italian to ensure that cultural and linguistic nuances were captured.

- The transferability of our findings to other settings is uncertain as all interviews were conducted in Italy and patients were recruited from four centres within Diaverum, a for-profit dialysis service provider.

\section{INTRODUCTION}

Patients on chronic dialysis experience significantly reduced quality of life and overall 
survival compared with the general population. ${ }^{1}{ }^{2}$ Haemodialysis therapy at home (any haemodialysis conducted at home by a patient or caregiver including frequent hours' haemodialysis and nocturnal home haemodialysis) is associated with substantially better survival $^{34}$ and may improve quality of life, compared with haemodialysis in a hospital or clinic setting, although adequately powered randomised trials are lacking. ${ }^{5} 6$ While the National Institute for Clinical Excellence guidelines recommend that all suitable patients are offered the choice between home haemodialysis or haemodialysis in a hospital or satellite unit, ${ }^{7}$ the global availability and use of home haemodialysis is very low. Home haemodialysis now represents $12-25 \%$ of all haemodialysis in Australia and New Zealand, ${ }^{1}$ whereas approximately only $1 \%$ of the nearly 400000 patients on haemodialysis in the $\mathrm{USA}^{8}$ and the 21000 patients on haemodialysis in the $\mathrm{UK},{ }^{9}$ perform their haemodialysis at home. Middle-income countries (annual per capita gross national income US\$3256-10 065) provide nearly no home haemodialysis. ${ }^{10}$

The reasons for highly variable global uptake in home haemodialysis remain incompletely understood. Previously identified barriers to home dialysis in the USA include inadequate dialysis service reimbursement or caregiver support, lack of innovative and patientfriendly dialysis equipment, competing business interests for dialysis providers, reliability of supplies and regulations that require frequent physician visits. ${ }^{11}$ Patients who are asked about self-care dialysis perceive that they have insufficient knowledge and skills to perform haemodialysis and are apprehensive about the unsupervised nature of home-based dialysis and social isolation. ${ }^{12}{ }^{13}$ While more is known about the perceptions and expectations of patients who have home haemodialysis as an active treatment choice, the perceptions and beliefs held by patients and caregivers who have little experience or opportunity of home haemodialysis are less well understood and greater understanding of them may help clinicians and providers develop successful and sustainable home haemodialysis programmes in regions without established home-based services.

This study aims to explore the beliefs and attitudes of patients and caregivers about home haemodialysis in a region with very low rates of home haemodialysis use.

\section{METHODS}

Participants were eligible if they were an adult receiving in-centre haemodialysis or an identified caregiver who had not received specific education about home haemodialysis and for whom home haemodialysis was not an available treatment option. We defined caregiver as any adult person (eg, spouse, close friend or family member) who provided care or support on a regular basis for a person on haemodialysis. We purposively selected participants to ensure that we captured the perspective of patients from a range of age, access to a potential caregiver, duration of in-centre haemodialysis and interest in home haemodialysis as well as both genders and caregivers with a range of age, years of caring and relationships to patients and both genders. We specifically included some patients who might be eligible for home haemodialysis. All participants were recruited by their primary physician from four centres within Diaverum, a large dialysis provider in Europe, Australia and South America. The centres were located in four Italian cities (Bari, Marsala, Nissoria and Taranto) that do not currently provide home haemodialysis services.

We developed an interview guide based on a literature review on home haemodialysis ${ }^{12}{ }^{14-22}$ and on discussion among the research team (AT/SP/GS/JC). Two weeks before the interview, participants were given a brochure on home haemodialysis (provided in online supplementary Digital Content 1) and invited to ask questions of the research team. Before the interview, participants were provided standardised verbal and written information about home haemodialysis that described the range of potential dialysis modalities (peritoneal dialysis, in-centre haemodialysis and home haemodialysis, including frequent and/or nocturnal schedules). The in-depth interview questions and prompts are provided in boxes 1 and 2. In October and November 2011, trained qualitative researchers (MR and LG) conducted face-to-face semistructured interviews with patients and caregivers in Italian, the native language of both the interviewers and participants. Recruitment was ceased when theoretical saturation was reached, defined as the time point in which little or no new concepts arose from subsequent interviews. Contextual details were recorded after each interview. All interviews were digitally audio-recorded and transcribed verbatim in Italian language.

The transcripts were entered into HyperRESEARCH (ResearchWare Inc USA V.3.0.2), a software package for storing, coding and searching qualitative data. Drawing on grounded theory and thematic analysis, ${ }^{23}{ }^{24} \mathrm{MR}$ and LG coded the transcripts line-by-line in the Italian language. They then independently identified concepts inductively and grouped similar concepts relating to patient and caregiver beliefs and expectations of home haemodialysis. The concepts, descriptors and supporting quotations were translated by MR and LG into English for discussion. Through a series of discussions, three authors (MR/LG/AT) reviewed and refined the coding scheme to develop themes that captured all concepts relating to patient and caregiver perspectives on home haemodialysis. To enhance the analytical framework and potential for offering meaningful insight, the thematic schema were then discussed within the research team.

\section{RESULTS}

Twenty-two haemodialysis patients and 20 of their identified caregivers participated in the study. All participants agreed to participate and their characteristics are 


\section{Box 1 Semi structured interview questions and topic guide—patient interview}

- Brief introduction of the study and obtain informed consent (to introduce and provide context)

- General experiences of dialysis

What were your initial thoughts or reactions when you were told you needed dialysis? What about now?

How do you feel when you go on dialysis and how do you feel between dialysis sessions?

How has your life changed since you started dialysis?

What is the most difficult or challenging thing about dialysis? How do you cope with it?

Do you ever miss dialysis treatments, why? Does missing dialysis impact your well-being, how?

What are your life priorities at the moment? Have they changed since before you started dialysis?

- Social support

Knowledge: Did you know about the different dialysis treatment options before starting dialysis? (peritoneal dialysis and haemodialysis) How did you come to know about them? Do you feel you received enough information about dialysis before you started dialysis, why?

Decision making: Which one do you think is better, why? If you were given the option between haemodialysis and peritoneal dialysis, why did you choose your current dialysis treatment?

Outcome preferences: If you could improve dialysis, what sorts of things would you change about dialysis? Imagine you had two types of dialysis to assess, what sorts of things would you measure or assess to determine which dialysis was better? (eg, survival, hospitalisation, dialysis adequacy, anaemia, blood pressure, nausea, cramps, headaches, quality of life-social freedom, depression, anxiety, employment control, energy, flexibility, ability to travel)

- Healthcare services

How happy are you with the care and treatment you are receiving in the hospital, why?

If you were in charge of this dialysis unit, what would you change to improve the experiences of dialysis patients?

- Home haemodialysis

Knowledge: what have you heard about home haemodialysis?

What would be the main difference for you between home haemodialysis and in-centre haemodialysis? (focus on impact on lifestyle, health and self-perception)

Is there anything else about home dialysis you would like to know about?

- *Provide brief description about home haemodialysis

Attitudes: Would you like to consider having haemodialysis at home? Why?

Perceived benefits: Are there any benefits of home haemodialysis compared with your current dialysis treatment? (freedom, flexibility, convenience, simplicity, effectiveness, dietary freedom, less waiting at hospital for dialysis, less travel time, ability to work or socialise, more family involvement, more privacy)

Perceived risks: What do you see are the risks of doing haemodialysis at home? (isolation, risk/safety, family burden, home modification)

Facilitators: What sorts of programmes or support do you think would help you to consider doing home haemodialysis? (plumbing, education, support, group meetings and emergency contact)

Support: Do you know of someone who would help you if you did dialysis at home? Have you discussed with anyone about it, if so, what do they think about home dialysis?

provided in table 1. Each interview lasted between 20 and $90 \mathrm{~min}$. Patients were aged between 34 and 78 years and the duration of dialysis ranged from 3 months to 26 years. Approximately half of the patients ( $\mathrm{n}=9$ $(41 \%))$ had a family caregiver and all except for one lived within a $30 \mathrm{~min}$ drive to the dialysis centre. Caregivers were aged between 38 and 78 years; most were women $(n=17(85 \%))$ and more than half $(n=12$ $(60 \%))$ were in a spousal relationship with the patient.

We identified three themes reflecting positive attitudes towards home haemodialysis: flexibility and freedom (increased autonomy, minimised wasted time, liberation from strict dialysis schedules and gaining self-worth); comfort in familiar surroundings (family presence and support and avoid the need for dialysis in hospital) and altruistic motivation. We identified four themes that encompassed negative attitudes: disrupted sense of normality; family burden (an onerous responsibility, caregiver uncertainty and panic and visually confronting); housing constraints and reliance on professional healthcare (relinquishing security, isolation from peer support and satisfaction with in-centre services). A thematic schema is provided in figure 1. Illustrative quotations to illustrate each theme are provided in table 2 . Quotations are also available in the original Italian language (see online supplementary Digital Content 2).

\section{Positive themes}

Flexibility and freedom

Increased autonomy-The participants were aware they could choose their own treatment schedule with home haemodialysis, which meant that they would be able to continue to work and participate in other day-to-day activities by scheduling dialysis away from these commitments. Some respondents believed that home haemodialysis would be particularly suited to younger patients, providing them with the opportunity to manage their multiple family, social and work roles better and increase their availability for employment. 


\section{Box 2 Semi structured interview questions and topic guide—caregiver interview}

- Brief introduction of the study and obtain informed consent (to introduce and provide context)

- General experiences of caregiving

How long have you known your family member on dialysis?

How has your life changed since your family member had to go on dialysis?

What is the most difficult or challenging thing about caring for someone on dialysis? How do you cope with it?

How do you feel about your situation and is there anything you can do to improve your situation?

What are your life priorities at the moment? Have they changed since before caring for your loved one on dialysis?

Do you have any health problems yourself? Can you describe these and how you manage your own health as well as the well-being of your family member on dialysis?

- Healthcare services

How happy are you with the care and treatment your family member is receiving in the hospital, why?

If you were in charge of this dialysis unit, what would you change to improve the experiences of dialysis patients or family caregivers?

- Home haemodialysis

Knowledge: what have you heard about home haemodialysis?

What would be the main difference for you, as a caregiver, between home haemodialysis and in-centre haemodialysis? (focus on impact on lifestyle, health and self-perception)

Is there anything else about home dialysis you would like to know about?

- *Provide brief description about home haemodialysis

Role: What do you think your role would be if your family member did dialysis at home? What might be the biggest challenge in helping someone with doing dialysis at home? Do you think you would be able to cope with these roles and responsibilities?

Attitudes: Would you like to consider having your family member do haemodialysis at home? Why?

Perceived benefits: Are there any benefits of home haemodialysis compared in-centre dialysis for you as a caregiver? (freedom, flexibility, convenience, simplicity, effectiveness, dietary freedom, waiting at hospital for dialysis, less travel time, ability to work or socialise, more family involvement and more privacy)

Perceived risks: What do you see are the risks of doing haemodialysis at home? (isolation, risk/safety, family burden and home modification)

Barriers: What do you foresee might be practical problems with home haemodialysis?

Facilitators: What sorts of programmes or support do you think would help you to consider doing home haemodialysis? (plumbing, support, group meetings and emergency contact)

Support: Do you know of someone who would help you if you did dialysis at home? Have you discussed with anyone about it, if so, what do they think about home dialysis?

Outcome preferences: If you could improve dialysis, what sorts of things would you change about dialysis? (travel time and energy)

Minimise wasted time-Both patients and caregivers anticipated that they would not have to waste time travelling while on home haemodialysis, which would give them additional freedom to do 'normal' activities. Patients were particularly frustrated about waiting in the dialysis clinic before starting dialysis and waiting for transport to return home.

Liberation from strict dialysis schedules-Some patients felt that adhering to time-intensive and rigid dialysis schedules was stressful and burdensome. Patients felt physically and psychologically burdened by the rigid constraints of in-centre haemodialysis schedules and expected that home haemodialysis would offer increased flexibility and freedom. With home haemodialysis, the participants felt they would no longer be 'mentally obliged' to attend their scheduled dialysis session in-centre.

Gaining self-worth-Some participants believed that doing home haemodialysis would allow them to provide better support for their family. One patient, who was a father, felt that home haemodialysis would allow him to work more and better provide for his family financially.

\section{Comfort in familiar surroundings}

Family presence and support-Home haemodialysis was perceived by patients and caregivers as a preferred option for some patients who desired to be in their own home or with their family while having treatment. Some caregivers also felt that patients would feel more relaxed and comfortable at home with their family when on dialysis.

Avoid the need for dialysis in 'hospitalisation'-Some participants felt they would prefer being at home rather than in clinic, because at the clinic they were surrounded by 'sickness'. At home, they believed they would be in better 'frame of mind'. Patients expressed that they wanted to have treatment in their own bed and did not want to see and interact with other sick patients, which some thought 'magnified' their own disease identity.

\section{Altruistic motivation}

A few patients expressed a keenness to help others and believed that they would consider home haemodialysis to set an example for other patients. One patient believed that haemodialysis offered a new opportunity for patients to be actively involved in their own care. Another patient, who was also a physician, felt that starting home haemodialysis himself would 'spur the authorities' to consider offering home haemodialysis as an option. 


\section{Table 1 Participant characteristics}

\begin{tabular}{|c|c|}
\hline Characteristics & Interviewees (n (\%)) \\
\hline \multicolumn{2}{|l|}{ Patients $(n=22)$} \\
\hline \multicolumn{2}{|l|}{ Gender } \\
\hline Male & $16(73)$ \\
\hline Female & $6(27)$ \\
\hline \multicolumn{2}{|l|}{ Age } \\
\hline 30-39 & $1(5)$ \\
\hline $40-49$ & $5(23)$ \\
\hline $50-59$ & $5(23)$ \\
\hline $60-69$ & $7(31)$ \\
\hline $70-79$ & $4(18)$ \\
\hline \multicolumn{2}{|l|}{ Time on dialysis } \\
\hline$<1$ year & $3(14)$ \\
\hline $1-5$ years & $11(50)$ \\
\hline $6-10$ years & $4(18)$ \\
\hline$>10$ years & $4(18)$ \\
\hline \multicolumn{2}{|l|}{ Vascular access } \\
\hline Central venous catheter & $2(9)$ \\
\hline Fistula & $20(91)$ \\
\hline \multicolumn{2}{|l|}{ Has a family caregiver } \\
\hline Yes & $9(41)$ \\
\hline No & $13(59)$ \\
\hline \multicolumn{2}{|l|}{ Travel time to dialysis centre } \\
\hline$<10 \min$ & $3(14)$ \\
\hline $10-30 \mathrm{~min}$ & $18(81)$ \\
\hline $31-60 \mathrm{~min}$ & $1(5)$ \\
\hline \multicolumn{2}{|l|}{ Caregivers $(n=20)$} \\
\hline \multicolumn{2}{|l|}{ Gender } \\
\hline Male & $3(15)$ \\
\hline Female & $17(85)$ \\
\hline \multicolumn{2}{|l|}{ Age (years) } \\
\hline $30-39$ & $1(5)$ \\
\hline $40-49$ & 7 (35) \\
\hline $50-59$ & $4(20)$ \\
\hline $60-69$ & 7 (35) \\
\hline $70-79$ & $1(5)$ \\
\hline \multicolumn{2}{|l|}{ Years as a caregiver } \\
\hline$<1$ & $1(5)$ \\
\hline $1-5$ & $12(60)$ \\
\hline $6-10$ & $3(15)$ \\
\hline$>10$ & $4(20)$ \\
\hline \multicolumn{2}{|l|}{ Relationship to patient } \\
\hline Spouse or partner & $12(60)$ \\
\hline Parent & $1(5)$ \\
\hline Child & $6(30)$ \\
\hline Sibling & $1(5)$ \\
\hline
\end{tabular}

\section{Negative themes}

\section{Disrupted sense of normality}

Living with a haemodialysis machine at home was perceived by some patients to be an unrelenting reminder of their disease. Some participants felt they would be unable to escape their disease and medical burden on home haemodialysis.

\section{Family burden}

An onerous responsibility-Participants, both patients and caregivers, believed that home haemodialysis would be an overwhelming responsibility for family caregivers. They felt it would be unjustifiable and unfair to 'enslave' caregivers with the physical and emotional burden of dialysis. They thought that if a caregiver had to be present during the hours they were on dialysis, caregivers would therefore have to make significant personal sacrifices impacting their work and social lives. Some caregivers expressed apprehension about taking on the responsibility of dialysis and believed they would be afraid or unable to see their loved one 'suffer' while on the dialysis machine. One caregiver stated that they did not want to be blamed and scolded by the patient if they experienced a problem with the dialysis machine.

Caregiver uncertainty and panic-Many caregivers felt inadequate, apprehensive and unable to perform dialysis care and manage complications. For example, caregivers were afraid of needles and felt unable to assist with cannulation. Caregivers anticipated they would be incapacitated by panic and unable to help the patient if they encountered a technical problem or complication. Even with training, caregivers doubted they could successfully manage medical responsibilities which they perceived belonged to professional medical healthcare providers who had necessary expertise, training and experience.

Visually confronting-Some patients believed that having the haemodialysis machine at home would be too confronting for their family. For example, they thought family members would feel distressed at the sight blood and needles, and that home haemodialysis would be particularly 'traumatic' for young children.

\section{Housing constraints}

Both patients and caregivers believed that home haemodialysis would limit their living space and they were therefore reluctant to consider having a haemodialysis machine in their living space. Participants also thought that home haemodialysis would not be feasible because they had inadequate room to accommodate a machine at home. In addition, patients were concerned that they would be unable to make the necessary plumbing or electrical modifications for a dialysis machine if they did not own their home. Some felt that they were unable to ensure sufficiently sterile conditions for home haemodialysis.

\section{Healthcare by 'professionals' not 'amateurs'}

Relinquishing security-Participants appreciated that physicians and nurses had more experience and expertise, which provided them with a sense of reassurance. They believed staff could remain composed and manage emergencies or complications if they occurred. For home haemodialysis, some participants were worried they might be left alone in managing their dialysis treatment.

Satisfaction with in-centre services-Some caregivers believed patients were contented with the quality of care received in the centre. They thought services were efficient and described the centre as having a 'comforting 
Figure 1 Thematic schema of patient and caregiver beliefs and expectation about home

haemodialysis.

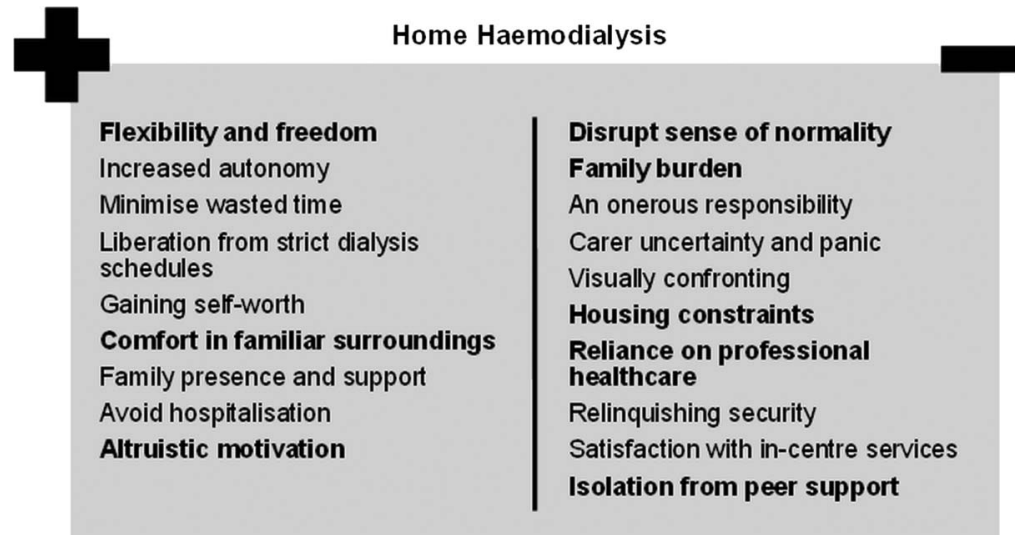

and familiar atmosphere'. Most participants also lived in close proximity to the centre and transport was coordinated and paid for by the dialysis provider. Therefore, they had little motivation or 'reason' to opt for home haemodialysis instead of in-centre haemodialysis.

\section{Isolation from peer-support}

Caregivers and patients felt that patients benefitted from the social support and camaraderie they received from staff and patients in the clinic. They believed that home haemodialysis meant losing contact with patients and staff, whom they relied on for support to cope with their disease.

\section{DISCUSSION}

This study highlights positive (flexibility and freedom, comfort in familiar surroundings and altruism) and negative (disrupted sense of normality, family burden, housing constraints and healthcare by 'professionals' not 'amateurs') patient and caregiver perspectives that are relevant when considering patient acceptance and uptake of home haemodialysis in regions without established home-based haemodialysis care. Patients and caregivers who have no direct experience of home haemodialysis believe that home haemodialysis can offer them more flexibility, freedom and time for family and work and provide physical and mental liberation from strict in-centre haemodialysis schedules. Some perceive that starting home haemodialysis is an opportunity to be an 'exemplar' for other patients, which might promote the wider acceptance and uptake of home haemodialysis by others.

However, despite the potential advantages of home haemodialysis, patients and caregivers in these regions are very concerned that home haemodialysis will be an overwhelming physical and emotional burden on caregivers and families. Patients and caregivers lack confidence that they have sufficient expertise and emotional fortitude to accept the medical responsibility of managing dialysis and its potential complications. They believe that performing home haemodialysis means relinquishing a sense of security provided in a supervised dialysis unit and will isolate them from the support of clinicians and their peers. Caregivers, in particular, believe that patients are contented with the quality of care received in dialysis centres and would not consider home haemodialysis.

Our findings in patients on in-centre haemodialysis and their caregivers in regions without home haemodialysis services are remarkably coherent with those reported by patients established on in-centre haemodialysis in other clinical settings, including in regions that have active home haemodialysis programmes. An observational study of individuals on in-centre haemodialysis who would be eligible for nocturnal home haemodialysis found that such patients had little interest in starting nocturnal home haemodialysis (haemodialysis at home for 6-8 h, 4-6 nights/week) due to lack of confidence in performing nocturnal haemodialysis and selfcannulation, concerns over lower quality of care and fears of catastrophic clinical events on home haemodialysis. ${ }^{12}$ In a survey of in-centre haemodialysis patients, prominent reasons for not choosing self-care dialysis (peritoneal dialysis and home and self-care haemodialysis) similarly included a belief patients should not be unsupervised on haemodialysis, fear of social isolation from peers, perceived lack of efficacy in performing selfcare and needle phobia. ${ }^{25}$ Previous research has shown that maintaining the status quo is an important factor ancing treatment alternatives, patients may be reluctant to change to a new treatment modality even when potential advantages are identified and recognised. This phenomenon is observed even in patients on in-centre haemodialysis in areas with relatively high home haemodialysis use, such as Australia, ${ }^{26}$ and may explain why patients already established on in-centre haemodialysis across multiple settings identify strong and consistent barriers to home haemodialysis regardless of the availability of home haemodialysis services to them.

Our findings have important implications for successful initiation and implementation of new home haemodialysis programmes in regions without established services. It appears that regardless of whether patients have access to home haemodialysis services, patients who have become established on conventional in-centre when patients choose a dialysis modality. ${ }^{13}{ }^{14}$ When bal- 
Table 2 Illustrative quotations translated from original Italian language to illustrate themes*

\section{Themes} Quotations (English)

Flexibility and freedom

Increased autonomy

"Just to avoid having to come here [in the clinic], and not to have a time restrictions. On reflection, doing home haemodialysis, I could manage my time as I prefer." (47M Patient)

"I think, I don't know if it's possible, that you can do dialysis while you are at home and have nothing else to do, and for someone who has a job this is absolutely the best solution. If I' $\mathrm{m}$ busy during the morning I will not do dialysis in the morning but I can do dialysis at lunch time, rather than lose an opportunity to work in the morning. On the contrary, here [in the clinic] I cannot say 'I'm busy today, I will come for dialysis in the afternoon or this evening." (51M Patient)

"I welcome home haemodialysis, because I think that is very good for young people. You can manage better your things; it gives more freedom, not only time. You can decide to do 6 or 8 hours of dialysis, connecting at midnight and disconnecting at 6 in the morning and go to work." (41M Patient)

Minimise wasted time

"The time I waste having to come here in the dialysis clinic, the waiting time [before you can start dialysis.]" (63M Patient) "Sometimes you have to wait half an hour, even longer before you start dialysis, say at night, to be connected to the monitor. The driver comes to pick me up at 5.10 or 5:15 and I'm connected at 6.45...then I come home late at night." (64M Patient) "Someone who does dialysis wants to go home quickly, they don't want to wait. My husband [doing in-centre haemodialysis] wants to go home immediately, he does not want to wait. He does not want to wait half an hour, 5 minutes are fine, but no more. Sometimes they have wait until it is decided which driver they will go back home with, whether it is this car or the other car. 20 minutes is too much." (78F Caregiver)

"At home he is in his environment, he does not have to wait, that's the thing; that he should not have to wait for dialysis." (79F Caregiver)

Liberation from strict dialysis schedules "The only advantage that I can see is psychological. I will not be in a hurry to get ready in the morning. I can be more comfortable with my own timetable." (49M Patient)

"At home he is in his environment, he does not have to wait, that's the thing; that he should not have to wait for dialysis." (79F Caregiver)

"You are not mentally obliged to come here [in the clinic] in the morning...you feel psychologically freer." (64M Patient)

"Autonomy is very important for him because he can decide to do dialysis when it is most convenient for him...he can decide independently. It is really for him, for our family it is OK, we have made arrangements. He is the one who has this need for more flexibility in shifts, for him this is a very limiting issue that he has to come here during those 3 days at a specific hour." (47F Caregiver)

Gaining self-worth

"My work is the only income to support my family, because I am a husband and a father and I must take care of my family, I wish they are always fine. So I am the only person in charge. If I should die, I am worried about this, and this makes me feel nervous, of course...because when you no longer have a good income, you cannot buy what is needed [for the family]." (51M Patient)

Comfort in familiar surroundings Family presence and support

"The comfort to be at home, being close to your family, and if you do not feel well they take care of you...I must confess that when my wife comes in the clinic, and she comes often, I am really happy." (51M Patient)

"These family moments, this extra time you can spend with family. Doing it at home for her would be like a game because living with my children she would play with my son, with my daughter. If someone buzzes at the door, she can ask you who it is. Then if the phone rings she can spend time with someone on the phone. She sometimes carries a mobile phone here in the clinic so she can chat with someone, that's how she spends her time." (40F Caregiver)

"For her stay at home, in her environment, is everything to her. For her to go away from home is torture...I do not know the right words to express this feeling she has." (52F Caregiver) 


\section{Themes}

Avoid hospitalisation

Altruistic motivation

Disrupt sense of normality

\section{Quotations (English)}

"And then, if he is with his family most of the time, he is more serene, even psychologically. For him it is not so much the physical pain or what we say, the dialysis, the issue is he does not want to be away from home." (47F Caregiver)

"You'd feel freer I think, staying at home, in your environment where you live. While now you are in an environment where you see other sick people. Your frame of mind is much better [at home]." (50M Patient)

"You are on your own bed, no contact with other people; you don't have to see the other's sickness, which makes you think of your own sickness, and magnifies it." (76M Patient)

"Thinking also of other patients who are my friends in dialysis, young or old people; I'm inclined to do a thing like that [home haemodialysis] for them. Why not? It is a new experience, an experience of life. At least for once, we as patients will be actively involved. When I didn't have all these problems I worked in first aid, I like helping people...now I am in very bad health conditions, but l'd like to help other patients, not only for myself." (41M Patient)

"The future of dialysis is at home, and we will be the precursors, or at least we will spur the authorities to take in serious consideration the possibility to do HHD." (76M Patient)

"Keeping the dialysis monitor at home would be a disadvantage. Because it would be a psychological burden, you know, to see the monitor each moment of the day in your home." (65M Patient)

"I feel I am a free and normal man, but if I should have that machine at home, it wouldn't be the same, I'd always remember my sickness." (29M Patient) An onerous responsibility

"This [in-center haemodialysis] seems to have the lowest impact on my life, on my family members, and have the greatest return in terms of benefit and cost of living....Should I hold one of my family members to be with me 4 hours a day? Absolutely no." (48M Patient)

"Second, I would enslave my family for at least 4 hours, 4 hours next to a machine." (48M Patient)

"I cannot oblige a person, no matter how much she loves me, and I love her, to force her to stay with me during the treatment. If I could do it alone, ok. But I can't stand to have a person make a sacrifice for me." (49M Patient)

"My wife, my daughter should learn, but they are not always available, they work all the day. You need a person at home with you, always." (62M Patient)

"Because if he does [dialysis] at home then I cannot even go out because I have to be following him." (60F Caregiver)

"The caregiver, who does not have your disease, all the sudden has to take this burden as their own. You involve him in an incredible way; you make him bear your disease." (49M Patient)

"I do not have time to do it because l've got many other things to do." (48M Caregiver)

"It takes a lot of patience because you should devote one day, a bad day, with him, I should always be with him...I should leave my job, there are reasons which would not allow me to do this." (62F Caregiver)

"It is like I said, it is an issue of caregiver competence, an issue of professionalism. My husband always tells me that in the dialysis unit, they monitor blood pressure constantly. If there are issues they immediately act upon them. How could I do this? I would be very scared, I would feel like I have the burden of a big responsibility." (62F Caregiver)

"I cannot stand seeing someone suffer." (62F Caregiver).

"No absolutely not. I'm already shaking." (40F Caregiver)

"I am a very apprehensive, anxious, I'm afraid to make mistakes so I would be very scared to make mistakes and not understand things." (62F Caregiver)

"In the event of complications, I am powerless. I cannot do anything. I do not have the training of a nurse or a doctor, so if anything should happen, any complications, I wouldn't know what to do." (64F Caregiver)

"Something could happen and I would be panicking." (49M Caregiver) 
"First, because when someone does a dialysis treatment, they need to have a trained professional person near them." (64 F Caregiver)

"[Home haemodialysis] makes no sense to me because it takes a lot of expertise. Also because of my husband's nature, we would end up arguing all the time because he is suspicious of people, my husband would blame me if I could not handle it well at home. He always fears others will do the wrong thing." (62F Caregiver)

Visually confronting

"First thing I said honestly, was I will never put those needles in your arm! (48F Caregiver)

traumatic." (34F Patient)

Housing constraints

"I don't like to have to limit the space in my house." (48M Patient)

"You should make the room available for the machine, the supplies...it can't be at home." (76F Patient)

"I haven't got the room, how can I do? You must have a bed for the treatment, how can I do? (59M Patient)

"It is a structural problem. If you live in a rental apartment you cannot make all these modifications to the house, even if small, for piping, etc." (50M Patient)

"I have not got a sterile room. You need a clean room [for home haemodialysis]. You need to put the machine, the appropriate bed." (65F Caregiver)

"It would be a hassle for me as well. You have to change the house, I should have to redo piping. tubing, facilities." (56M Caregiver)

"You need a big house, a room only for you. Other people [in the family] cannot enter in that room while you have your things there. Moreover, I have a dog at my place! (58F Patient)

"There may be technical problems, such as the dialysis machine, for example the electricity goes off, I mean, while he is doing

Healthcare by 'professionals' not 'amateurs' dialysis, what can I do? The thing is I do not have an electricity generator set." (70F Caregiver)

Relinquishing security

"It is a question of safety. Here I have long term specialized doctors and paramedics who have years or decades of experience, which would certainly not be reproducible at home. If an emergency happens, here the staff are well trained. They don't need someone to tell them what to do, they do not panic." (49M Patient)

"There are always risks, there may be risks even here, but here I can feel more confident. I've got service, there are doctors and nurses. They know exactly what to do in case of bleeding for example, but at home, I would know what to do but I haven't all the supplies. Here you have everything." (76M Patient)

"At home you may be happier but all this fades when worries take over. The serenity of staying at home is over. If the caregiver can't come to help me for that treatment, what do I do? I will become worried, who should I call?" (49M Patient) "Yes, yes. You know what? I am calmer, more serene, peaceful, because there are the doctors that if anything happens, they are already here. If he feels bad the doctors and nurses never leave him. That is a very different from home. At home I can leave, go to buy groceries, I find that is not impossible (47F Caregiver)

"I'm interested in security, specialization and the professionalism of a person who is putting his hands on a patient like this." (47M Caregiver)

Satisfaction with in-centre services
"She is happy in the clinic compared to the hospital; because in the past two or three times we went to the hospital, we saw the difference, there is no comparison of the situation, the level of care here is really better." (47M Caregiver

"This is his second home. They are exceptional. He calls [clinic staff] 'my angels'. We found a particular organization. So even if he had the benefits of a home dialysis, he would never accept that because he relies on all of them. He has that comforting, familiar atmosphere. They are all truly exceptional." (62F Caregiver) 


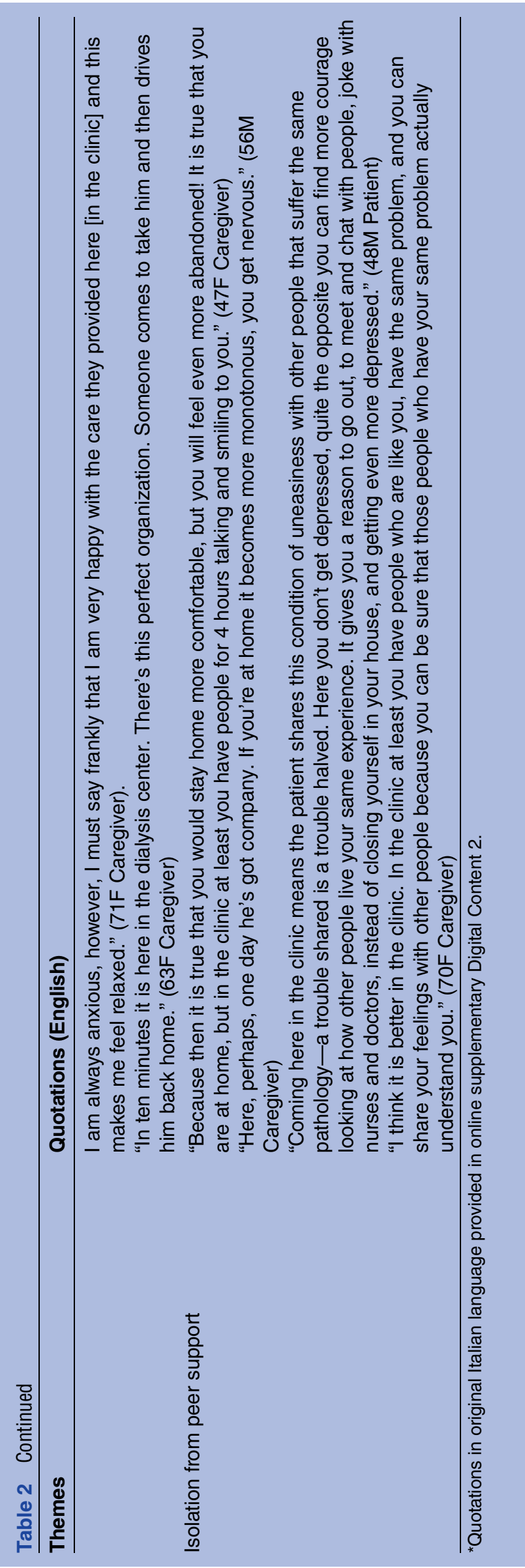

haemodialysis may be largely negative about home haemodialysis and view it as confronting and lack confidence in their ability to learn the technique. In contrast, patients experienced in home haemodialysis in other regions may be more positive and enthusiastic about home haemodialysis as a treatment choice and report generally high levels of satisfaction. ${ }^{15}{ }^{16}$ This disparity in attitudes and perceptions of home haemodialysis associated with current treatment modality suggests that discussions about treatment options for end-stage kidney disease that include home haemodialysis should be tailored to the existing haemodialysis experiences of patients and their families, and such targeted education may increase the acceptability and subsequent uptake of home haemodialysis for all patients.

Consistent with this approach, earlier studies have shown that patients and their caregivers in the predialysis phase of care and who have received comprehensive predialysis information about all modalities of dialysis treatment (including conservative care) are more likely to choose a home-based dialysis modality over other treatment options. ${ }^{27-29}$ However, given that the majority of the small number of home haemodialysis patients in the USA has experienced in-centre haemodialysis before starting home therapy, it is likely patients and caregivers concerns about home haemodialysis can be overcome with intensive educational strategies. Accordingly, the effectiveness of education about home haemodialysis for patients currently treated with in-centre haemodialysis warrants further study. In addition, given the present study is conducted with patients and their families currently treated with dialysis, the effects of predialysis education on patient and caregiver treatment preferences in regions without established home haemodialysis also deserve further evaluation. We have generated potential strategies and recommendations to increase the acceptability of home haemodialysis among patients and family caregivers in table 3 based on our data and the existing literature. We acknowledge that the relevance, appropriateness and effectiveness of these strategies may be different between predialysis and prevalent dialysis patients. The educational and support interventions may be delivered by specific nurse specialists involved in predialysis care and education and included as a part of predialysis clinics. Moreover, we suggest establishing database management and communication between the team members to plan and implement educational strategies, offering group education with patients and families, providing video-based and online materials for patients and families and allowing patients and families to meet other patients who are established on home therapies.

To our knowledge, this is the first study to examine the expectations and beliefs about home haemodialysis among in-centre haemodialysis patients and their caregivers who have received no formal education, choice or experience of home haemodialysis. The key characteristics of the studied population, including mean age, 
Table 3 Strategies and recommendations to promote the acceptability of home haemodialysis among patients and family caregivers

\section{Strategy}

Education

Minimise intrusiveness of home haemodialysis

Provide support and respite for caregivers and family members

Maintain patient access to medical and technical support

Minimise social isolation

Promote self-efficacy

\section{Action plan}

- Discussions about treatment options for end-stage kidney disease that include home haemodialysis should be tailored to the existing haemodialysis experiences of patients and their families

- Meet other patients on home dialysis therapies (haemodialysis, nocturnal haemodialysis and peritoneal dialysis) to listen to their experiences

- Use home haemodialysis machines that are less conspicuous (small sized and portable) and require minimal home modifications

- Promote awareness about home haemodialysis machines that are smaller and easy to operate

- Provide practical tips on minimising the visibility of home haemodialysis within the home

- Increase awareness of home haemodialysis equipment that require minimal or no caregiver assistance

- Identify and implement a forum for patients and families/caregivers to discuss experiences and decisions (online/physical)

- Appoint a dedicated mental health worker in the clinical team

- Ensure access to social work services (caregiver respite and allowance)

- Network with caregiver or consumer organisations and identify respite and support services for caregivers and families

- Offer home visits as determined on an individual, need-by-need basis

- Provide $24 \mathrm{~h}$ availability (eg, hotline) of clinical and technical staff

- Organise forums or social events for patients and caregivers (eg, face-to-face, telephone and web-based contact)

- Consider set up of community homes for haemodialysis

- Use home haemodialysis machines that are intuitive and simple to configure, programme and disconnect

- Provide comprehensive and timely home haemodialysis education and training (eg, individual training, forums, question and answer sessions and print and multimedia resources)

- Encourage the importance and benefits of independence and self-care

- Recruit home haemodialysis 'patient exemplars'

- Facilitate opportunities to learn from patients who are successfully performing home haemodialysis proportion with various comorbidities, mean values of $\mathrm{Kt} / \mathrm{V}$, albumin, treatment time, number of treatments per week, haemoglobin, ferritin, calcium, phosphorus, calcium $\times$ phosphorus product, interdialysis body weight gain, mean arterial pressure and proportion of diabetics are similar to those reported in the Italian dialysis and transplantation registry annual report (available at www. ridt.org). All interviews were conducted and coded in Italian to ensure that cultural and linguistic nuances were captured. However, our study has some limitations that suggest caution. The transferability of our findings to other settings is uncertain as all interviews were conducted in Italy and patients were recruited from four centres within Diaverum, a for-profit dialysis service provider. Despite this, similar findings have been identified in other studies on patient-perceived barriers to home haemodialysis. ${ }^{12} 30$ Notably, we did not purposively capture perspectives from only patients who were eligible for home haemodialysis. It is possible that patients and their families who were most concerned about home haemodialysis would not have been eligible for this treatment option and may have intensified the negative themes we observed. However, the perspectives and attitudes we captured are similar to those of patients in other regions already on in-centre haemodialysis who are considered eligible for home-based haemodialysis. $^{12}$

As a result of scarce data, further research is required to assess whether specific education strategies and support networks will increase the preference for and acceptability 
of home haemodialysis for patients and their caregivers in our regions. Although patients on peritoneal dialysis report greater satisfaction with care than patients on in-centre haemodialysis, ${ }^{31}$ additional research would provide valuable insight into the relative satisfaction of patients on differing home based therapies (haemodialysis and peritoneal dialysis). Limited high-quality data are available on the effect of information and support strategies for caregivers of people with chronic kidney disease; additional development and evaluation of services that specifically support carers and families would be valuable. ${ }^{32}$ Recently, a randomised trial has shown that more frequent in-centre haemodialysis (6 times/week) may improve survival and cardiovascular outcomes compared with conventional (3 times/week) in-centre haemodialysis. ${ }^{33}$ Adequately powered randomised trials of home haemodialysis (long-hours, increased frequency or nocturnal) compared with in-centre haemodialysis would provide more information on the effects of home haemodialysis on survival and quality of life.

Increased utilisation of home haemodialysis has been strongly advocated to promote autonomy, self-sufficiency, and a sense of mastery in the management of end-stage kidney disease and may improve clinical outcomes and overall patient well-being. However, despite the potential for increased independence and treatment freedom with home haemodialysis, patients established on in-centre haemodialysis are generally negative about home haemodialysis and hold concerns about caregiver burden, treatment intrusiveness, and their inability to manage dialysis and its potential complications. It is likely these perspectives and attitudes toward home haemodialysis are common to all patients established on in-centre haemodialysis regardless of the availability of home services in their region. Individualised education and information for patients and their families approaching end-stage kidney disease is therefore needed before patients start dialysis. Specific education about home haemodialysis and opportunities to learn from the experiences of other home dialysis patients in the predialysis phase of care may increase patient acceptability and uptake of home haemodialysis in regions without home haemodialysis services. In addition, educational strategies that increase patient acceptance of home haemodialysis once they have commenced in-centre haemodialysis warrants investigation.

\footnotetext{
Author affiliations

${ }^{1}$ Centre for Kidney Research, The Children's Hospital at Westmead, Sydney, New South Wales, Australia

${ }^{2}$ Sydney School of Public Health, The University of Sydney, Sydney,

New South Wales, Australia

${ }^{3}$ Department of Medicine, University of Otago Christchurch, Christchurch, New Zealand

${ }^{4}$ Department of Medicine and Community Health Sciences, University of Calgary, Calgary, Canada

${ }^{5}$ Diaverum Scientific Office, Medical-Scientific Office, Diaverum Renal Services Group, Lund, Sweden

${ }^{6}$ School of Medicine, University of Queensland at Princess Alexandra Hospital, Brisbane, Queensland, Australia
}

${ }^{7}$ Diaverum Medical Office, Diaverum Renal Services Group, Lund, Sweden

${ }^{8}$ Diaverum Renal Services Group, Lund, Sweden

${ }^{9}$ School of Medicine, Hofstra Northshore LIJ, Great Neck, New York, USA

${ }^{10}$ Department of Clinical Pharmacology and Epidemiology, Consorzio Mario Negri Sud, Bari, Italy

${ }^{11}$ Diaverum Scientific Office, Lund, Sweden

${ }^{12}$ Department of Emergency and Organ Transplantation, University of Bari, Bari, Italy

Acknowledgements We would like to thank all the patients and caregivers who volunteered their time to participate in this study.

Contributors AT participated in the design of the study carried out the thematic analysis and drafted the manuscript. SP designed the study, participated in the thematic analysis and critical review of the first and subsequent manuscript drafts. BM and JC provided intellectual input into first and subsequent manuscript drafts and assisted with thematic analysis. MR and LG did the interviews, coded and analysed the data, and provided intellectual input into subsequent manuscript drafts. DWJ assisted with thematic analysis and provided intellectual input into primary and subsequent drafts. $\mathrm{JH}, \mathrm{MO}$ and SF provided intellectual input into subsequent manuscript drafts. GS conceived the study, obtained funding, assisted with study design and thematic analysis, provided intellectual input into subsequent manuscript drafts and is the guarantor. All authors, external and internal, had full access to all of the data in the study and can take responsibility for the integrity of the data and the accuracy of the data analysis.

Funding The study has been part-funded by an unrestricted grant from Diaverum Renal Services Group, a global provider of renal services. The study sponsor commissioned the work to an independent steering committee and had no role in study design; collection, analysis and interpretation of data; writing the report and the decision to submit the report for publication; AT is supported by the National Health and Medical Research Council Early Career Fellowship ID 1037162; SP is supported in part by an by an unrestricted grant from Amgen Dompé administered by the Mario Negri Sud Consortium, Italy; DJ has received research grants, travel sponsorships and consultancy fees from Baxter Healthcare and Fresenius Medical Care and is a current recipient of a Queensland Government Health Research Fellowship. JH is Chief Medical Officer at Diaverum, a global provider of renal services; GFMS is Senior Vice President Scientific Affairs at Diaverum, a global provider of renal services: No other relationships or activities that could appear to have influenced the submitted work.

Competing interests None.

Patient consent Obtained.

Ethics approval Ethics approval was sought from all participating centres according to Italian law by informing the ethics committees (Italian Republic. Determination of the Italian Medicines Agency of 20 March 2008 (in Italian). Official Gazette of the Italian Republic. General Series No. 76; 31 March 2008). All participants provided written voluntary informed consent.

Provenance and peer review Not commissioned; externally peer reviewed.

Data sharing statement No additional data are available.

\section{REFERENCES}

1. McDonald S, Excell L. Chapter 4. Method and location of dialysis. In Australia and New Zealand Dialysis and Transplant Registry (ANZDATA) Registry 2009 report. Adelaide: ANZDATA, 2009.

2. US Renal Data System. USRDS 2010 Annual Data Report: Volume Two Atlas of End-Stage Renal Disease in the United States. Bethesda, MD: National Institutes of Health, National Insitute of Diabetes and Digestive and Kidney Diseases; 2010.

3. Pauly RP, Gill JS, Rose CL, et al. Survival among nocturnal home haemodialysis patients compared to kidney transplant recipients. Nephrol Dial Transplant 2009;24:2915-19.

4. Woods JD, Port FK, Stannard D, et al. Comparison of mortality with home hemodialysis and center hemodialysis: a national study. Kidney Int 1996;49:1464-70.

5. Culleton BF, Walsh M, Klarenbach SW, et al. Effect of frequent nocturnal hemodialysis vs conventional hemodialysis on left ventricular mass and quality of life: a randomized controlled trial. JAMA 2007;298:1291-9. 
6. Rocco MV, Lockridge J, Beck GJ, et al. The effects of frequent nocturnal home hemodialysis: the frequent hemodialysis network nocturnal trial. Kidney Int 2011;80:1080-91.

7. Guidance on home compared with hospital haemodialysis for patient with end-stage renal failure: NHS National Institute for Clinical Excellence,London UK, 2002.

8. U.S. Renal Data System, USRDS 2009 Annual Data Report: Atlas of End-Stage Renal Disease in the United States. Bethesda, MD: National Institutes of Health, National Institute of Diabetes and Digestive and Kidney Diseases; 2009.

9. The United Kingdom Renal Registry. Chapter 4. UK ESRD Prevalent Rates in 2008: national and centre-specific analyses, 2009. http:// www.renalreg.com/Reports/2009.html (accessed 2 Jun 2011).

10. MacGregor MS, Agar JW, Blagg CR. Home haemodialysis-international trends and variation. Nephrol Dial Transplant 2006;21:1934-45.

11. Golper TA, Saxena AB, Piraino B, et al. Systematic barriers to the effective delivery of home dialysis in the United States: a report from the public policy/advocacy committee of the North American Chapter of the International Society for Peritoneal Dialysis. Am J Kidney Dis 2011;58:879-85.

12. Cafazzo JA, Leonard K, Easty AC, et al. Patient-perceived barriers to the adoption of nocturnal home hemodialysis. Clin J Am Soc Nephrol 2009;4:784-9.

13. Pipkin M, Eggers PW, Larive B, et al. Recruitment and training for home haemodialysis: experience and lessons from the nocturnal dialysis trial. Clin J Am Soc Nephrol 2010;5:1614-20.

14. Morton RM, Tong A, Howard K, et al. The views of patients and carers in treatment decision making for chronic kidney disease: systematic review and thematic synthesis of qualitative studies. $\mathrm{Br}$ Med J 2011;340:c112.

15. Morton RM, Tong A, Webster AC, et al. Characteristics of dialysis important to patients and family caregivers: a mixed methods approach Nephrol Dial Transplant 2011;26:4038-46.

16. Derrett S, Darmody M, Williams S, et al. Older peoples' satisfaction with home-based dialysis. Nephrology 2010;15:464-70.

17. Fadem SZ, Walker DR, Abbott G, et al. Satisfaction with renal replacement therapy and education: the American Association of Kidney Patients survey. Clin J Am Soc Nephrol 2011;6:605-12.

18. Qamar M, Bender F, Rault $R$, et al. The United States' perspectives on home dialysis. Adv Chronic Kidney Dis 2009;16:189-97.
19. Manns B, Johnson JA, Taub K, et al. Quality of life in patients treated with hemodialysis or peritoneal dialysis: what are the important determinants. Clin Nephrol 2003;60:341-51.

20. Lee A, Gudex C, Povlsen JV, et al. Patients' views regarding choice of dialysis modality. Nephrol Dial Transplant 2008;23:3953-9.

21. Cases A, Dempster M, Davies M, et al. The experience of individuals with renal failure participating in home haemodialysis: an interpretative phenomenological analysis. $J$ Health Psychol 2011;16:884-94.

22. Diaz-Buxo JA, Crawford-Bonadio TL, St Pierre D, et al. Establishing a successful home dialysis program. Blood Purif 2006;24:22-7.

23. Liamputtong P. Qualitative research methods. Melbourne: Oxford University Press, 2009.

24. Kvale S. Interviews. Thousand Oaks, CA: Sage Publications, 1996

25. McLaughlin K, Manns B, Mortis G, et al. Why patients with ESRD do not select self-care dialysis as a treatment option. Am J Kidney Dis 2003:41:380-5.

26. Kidney Health Australia. Consumer perspectives on dialysis: first national census. Melbourne. 2011. http://www.kidney.org.au (accessed 10 Jul 2012)

27. Ahmed S, Addicott C, Qureshi M, et al. Opinions of elderly people on treatment for end-stage renal disease. Gerontology 1999:45:156-9.

28. Gomez CG, Valido P, Celadilla O, et al. Validity of a standard information protocol provided to end-stage renal disease patients and its effect on treatment selection. Perit Dial Int 1999;19:471-7.

29. Morton RL, Snelling P, Webster AC, et al. Dialysis modality preference of patients with CKD and family caregivers: a discrete-choice study. Am J Kidney Dis 2012;60:102-11.

30. Manns BJ, Taub K, Vanderstraeten C, et al. The impact of education on chronic kidney disease patients' plans to initiate dialysis with self-care dialysis: a randomized trial. Kidney Int 2005;68:1777-83.

31. Rubin HR, Fink NE, Plantinga LC, et al. Patient ratings of dialysis care with peritoneal dialysis vs hemodialysis. JAMA 2004;291:697-703.

32. Tong A, Sainsbury P, Craig JC. Support interventions for caregivers of people with chronic kidney disease: a systematic review. Nephrol Dial Transplant 2008;23:3960-65.

33. Chertow GM, Levin NW, Beck GJ, et al. In-center hemodialysis six times per week versus three times per week. $N$ Engl J Med 2010;363:2287-300. 